\title{
Correlations of cardiac function with inflammation, oxidative stress and anemia in patients with uremia
}

\author{
HEPING ZHANG, LEI FAN, HUAWEI LIAO, LIRONG TU, \\ JIE ZHANG, DAN XU and JIANGCHAO FENG \\ Department of Renal Medicine, Affiliated Hospital of North Sichuan Medical College, \\ Nanchong, Sichuan 637000, P.R. China
}

Received April 14, 2019; Accepted October 3, 2019

DOI: $10.3892 /$ etm.2021.9681

\begin{abstract}
The present study aimed to analyze the associations of cardiac function with inflammatory cytokines, oxidative stress and anemia in patients with uremia. A total of 79 patients with uremia were selected, and among those, 43 cases were complicated with cardiac dysfunction (observation group) and 36 patients were not (control group). The levels of inflammatory cytokines [C-reactive protein (CRP), interleukin-6 (IL-6) and IL-10], oxidative stress indicators [malondialdehyde (MDA), oxidized low-density lipoprotein (OX-LDL) and advanced oxidation protein products (AOPP)], blood routine parameters [hemoglobin $(\mathrm{Hb})$, platelets (PLT), mean corpuscular volume (MCV)], and cardiac function [including cardiac output $(\mathrm{CO})$, cardiac stroke volume (SV), left ventricular ejection fraction (LVEF), left ventricular end-diastolic diameter (LVEDd) and left ventricular end-systolic diameter (LVESd)] were measured. There were no statistically significant differences in age, sex, body mass index, total cholesterol, low-density lipoprotein cholesterol and high-density lipoprotein cholesterol between the observation and the control group $(\mathrm{P}>0.05)$, but the observation group had significantly higher triglyceride levels and blood pressure, as well as longer duration of uremia compared with those in the control group $(\mathrm{P}<0.05)$. Furthermore, the levels of CRP, IL-6, IL-10, MDA, OX-LDL, AOPP, Hb, PLT and MCV in the observation group were significantly higher than those in the control group. In addition, regarding cardiac function, the observation group had a decreased CO, SV and LVEF and an increased LVEDd and LVESd compared with those in the control group. Furthermore, CRP $(r=-0.636, \mathrm{P}<0.01)$ and MDA $(\mathrm{r}=-0.705$, $\mathrm{P}<0.01)$ were negatively correlated with $\mathrm{LVEF}$, while Hb levels were positively correlated with $\mathrm{LVEF}(\mathrm{r}=0.732, \mathrm{P}<0.001)$. In conclusion, serum inflammatory cytokines, oxidative stress and
\end{abstract}

Correspondence to: Dr Jiangchao Feng, Department of Renal Medicine, Affiliated Hospital of North Sichuan Medical College, 63 Cultural Road, Shunqing, Nanchong, Sichuan 637000, P.R. China E-mail: jiangchaofeng81@163.com

Key words: uremia, inflammation cytokines, oxidative stress, anemia, cardiac function the degree of anemia are associated with cardiac insufficiency in patients with uremia.

\section{Introduction}

Uremia, also known as end-stage renal failure in the clinic, is particularly common in nephrology. In such patients, uremia is frequently complicated with myocardial damage, leading to cardiac insufficiency (1). The most common causes of cardiac insufficiency in uremic patients are hemodynamic changes and metabolic abnormalities, leading to increased mortality (2). In patients with uremia, significant hemodynamic changes and accumulation of toxins are present, and myocardial tissue cells frequently have systolic and diastolic dysfunction (3). More than half of the patients with uremia succumb to cardiac insufficiency (4). Uremic patients frequently have varying degrees of anemia and decreased production of erythropoietin (5). Uremia is not only a pathological reaction of the body's response to renal dysfunction but also an inflammatory reaction associated with chronic oxidation (6). A previous study has defined this as a uremia-associated inflammatory response and the degree of anemia is aggravated due to reduced production of erythropoietin and impaired iron mobilization caused by an excessive inflammatory response (7). The occurrence of cardiac dysfunction in patients with uremia is significantly associated with oxidative stress in the body and oxidative stress is also an important contributing factor to the occurrence of cardiac dysfunction (8). Significantly increased oxidative stress levels in patients with uremia are caused by increased production of toxins and impaired excretion function in end-stage renal failure. Excessive oxidative stress mainly leads to damage of the patient's vascular endothelial cells, thereby promoting the occurrence of cardiac insufficiency (9). However, the exact association of cardiac function with inflammatory cytokines, oxidative stress and anemia in patients with uremia remains to be fully elucidated. The aim of the present study was to assess the association of cardiac function with inflammatory cytokines, oxidative stress and anemia in patients with uremia.

\section{Patients and methods}

Patients. A total of 79 patients diagnosed with uremia at the Department of Nephrology of the Affiliated Hospital of 
Table I. Comparison of general characteristics between the observation group and control group.

\begin{tabular}{|c|c|c|c|}
\hline General data & Observation group $(n=43)$ & Control group $(n=36)$ & P-value \\
\hline Age, years (mean \pm SD) & $58.53 \pm 6.98$ & $57.03 \pm 9.61$ & 0.619 \\
\hline Sex, male/female (n) & $29 / 14$ & $20 / 16$ & 0.145 \\
\hline Disease course, years (mean $\pm \mathrm{SD}$ ) & $11.78 \pm 2.05$ & $6.79 \pm 3.83$ & 0.018 \\
\hline $\mathrm{BMI}, \mathrm{kg} / \mathrm{m}^{2}($ mean $\pm \mathrm{SD})$ & $18.88 \pm 2.93$ & $18.79 \pm 3.29$ & 0.475 \\
\hline $\mathrm{TC}, \mathrm{mmol} / \mathrm{l}($ mean $\pm \mathrm{SD})$ & $5.83 \pm 1.05$ & $5.45 \pm 1.02$ & 0.068 \\
\hline $\mathrm{TG}, \mathrm{mmol} / \mathrm{l}(\mathrm{mean} \pm \mathrm{SD})$ & $2.94 \pm 1.05$ & $2.07 \pm 0.79$ & 0.042 \\
\hline LDL-C, mmol/1 (mean \pm SD) & $3.43 \pm 0.97$ & $3.08 \pm 0.76$ & 0.588 \\
\hline HDL-C, mmol/l (mean \pm SD) & $1.53 \pm 0.54$ & $1.45 \pm 0.33$ & 0.081 \\
\hline $\mathrm{SBP}, \mathrm{mmHg}($ mean $\pm \mathrm{SD})$ & $165 \pm 28$ & $147 \pm 24$ & 0.039 \\
\hline $\mathrm{DBP}, \mathrm{mmHg}($ mean $\pm \mathrm{SD})$ & $99 \pm 18$ & $83 \pm 16$ & 0.041 \\
\hline
\end{tabular}

BMI, body mass index; TC, total cholesterol; TG, triglyceride; LDL-C, low-density lipoprotein cholesterol; HDL-C, high-density lipoprotein cholesterol; SBP, systolic blood pressure; DBP, diastolic blood pressure.

North Sichuan Medical College (Nanchong, China) between May 2016 and June 2017 were recruited. Among them, 43 patients had cardiac insufficiency (observation group) and 36 patients had no cardiac insufficiency (control group). The cohort comprised 49 males and 30 females aged 40-72 years with an average age of $57.78 \pm 12.09$ years. The diagnostic criteria for uremia were as follows: Glomerular filtration rate $(\mathrm{GFR})<10 \mathrm{ml} / \mathrm{min}$ or serum creatinine $>707 \mu \mathrm{mol} / \mathrm{l}$. The exclusion criteria were as follows: patients with systemic multiple organ failure, those complicated with serious infections, rheumatoid arthritis and other autoimmune diseases, those taking topical steroids or receiving blood purification or peritoneal dialysis treatment prior to diagnosis, or those with mental illness, with incomplete clinical data or refusing to provide informed consent. The present study was approved by the ethics committee of the Affiliated Hospital of North Sichuan Medical College (Nanchong, China; no. 20160102AHNSMCE) and informed consent from the subjects was obtained prior to the study.

Measurement of blood biochemical parameters. After fasting for solids and liquids for $10 \mathrm{~h}$ overnight, fasting peripheral blood was collected from all patients enrolled and centrifuged to obtain serum, which was used to measure total cholesterol (TC), triglyceride (TG), low-density lipoprotein cholesterol (LDL-C), high-density lipoprotein cholesterol (HDL-C), hemoglobin (Hb), platelets (PLT) and mean corpuscular volume (MCV) using an automatic biochemical analyzer (Hitachi; model no. HTCA3060).

Detection of the levels of inflammatory cytokines. The levels of inflammatory cytokines [C-reactive protein (CRP), interleukin-6 (IL-6) and IL-10] were measured through immunoturbidimetry (BK-400; Shandong Boke CNC equipment Co., Ltd.) following the manufacturer's protocol. The reagents were provided by Shenzhen Genrui Biological Technology Co., Ltd. The levels of malondialdehyde (MDA; cat. no. ab238537; Abcam), oxidized low-density lipoprotein (OX-LDL; cat. no. ab242302; Abcam) and advanced oxidation protein products (AOPP; cat. no. ab242295; Abcam) were determined by ELISA according to the manufacturer's protocol.

Assessment of cardiac function. Cardiac function was determined through measuring the cardiac output (CO), cardiac stroke volume (SV), left ventricular ejection fraction (LVEF), left ventricular end-diastolic diameter (LVEDd) and left ventricular end-systolic diameter (LVESd) by a full-time technician using a Vivid 6-dimension ultrasound instrument (GE Healthcare).

Statistical analysis. SPSS 19.0 software (IBM Corp.) was used for data processing. The results were expressed as the mean \pm standard deviation. Student's t-test was performed for comparison of differences between two groups and the Chi-square test was adopted for comparisons of enumeration data. Pearson correlation was applied for correlation analysis. $\mathrm{P}<0.05$ was considered to indicate a statistically significant difference.

\section{Results}

Comparisons of clinical characteristics. There were no statistically significant differences in age, sex, body mass index, TC, LDL-C and HDL-C levels between the observation group and the control group $(\mathrm{P}>0.05)$. However, the observation group had significantly higher TG and blood pressure levels, as well as a longer duration of uremia compared with the control group $(\mathrm{P}<0.05$; Table I).

Increased levels of inflammatory cytokines in patients with cardiac dysfunction. The peripheral serum levels of CRP, IL-6 and IL-10 in the observation group were all significantly higher than those in the control group ( $\mathrm{P}<0.05$; Fig. 1).

Elevated oxidative stress in patients with cardiac dysfunction. The levels of MDA, OX-LDL and AOPP in the observation group were significantly increased compared with those in the control group ( $\mathrm{P}<0.05$; Fig. 2). 


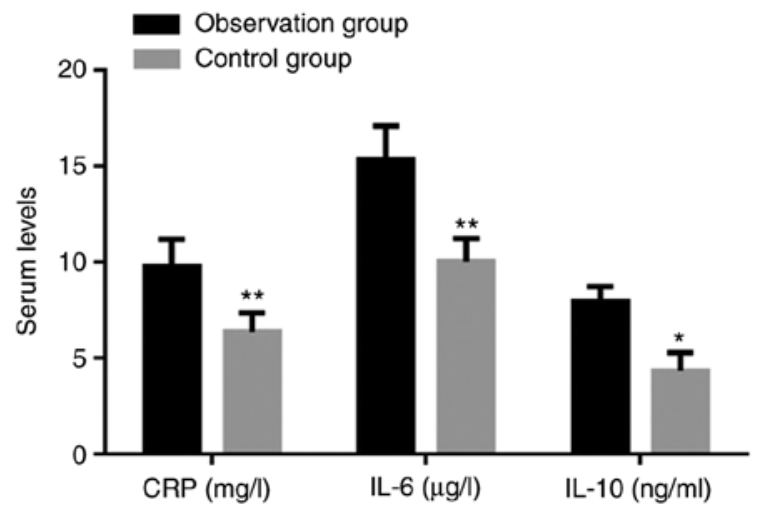

Figure 1. Levels of CRP, IL-6 and IL-10 in patients. Values are expressed as the mean \pm standard deviation and were analyzed by Student's t-test. ${ }^{*} \mathrm{P}<0.05$, ${ }^{* *} \mathrm{P}<0.01$, compared with the observation group. CRP, C-reactive protein; IL, interleukin.

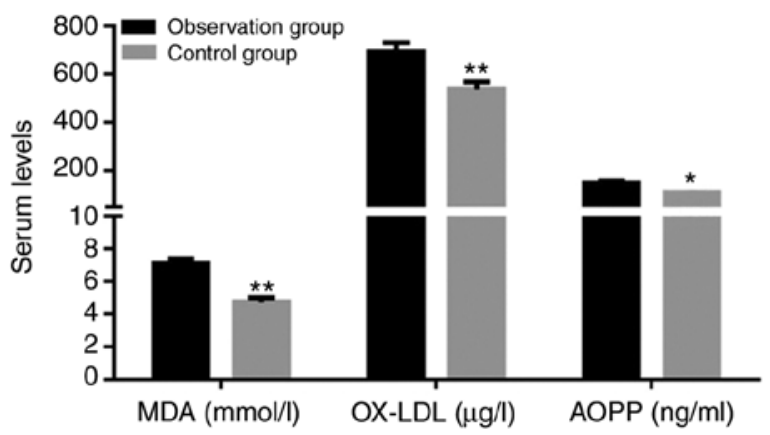

Figure 2. Levels of MDA, OX-LDL and AOPP in patients. Values are expressed as the mean \pm standard deviation and were analyzed by Student's t-test. ${ }^{*} \mathrm{P}<0.05,{ }^{* *} \mathrm{P}<0.01$, compared with the observation group. MDA, malondialdehyde; OX-LDL, oxidized low-density lipoprotein; AOPP, advanced oxidation protein products.

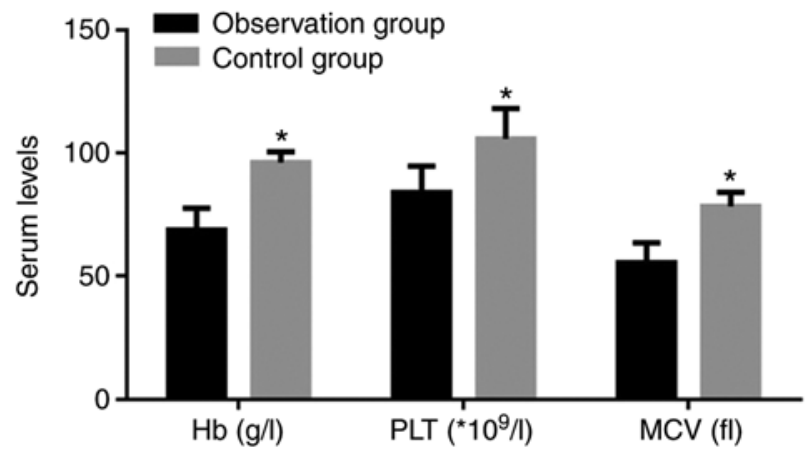

Figure 3. Levels of Hb, PLT and MCV. Values are expressed as the mean \pm standard deviation and were analyzed by Student's t-test. ${ }^{*} \mathrm{P}<0.05$, compared with the observation group. Hb, hemoglobin; PLT, platelets; $\mathrm{MCV}$, mean corpuscular volume.

Comparison of anemia. Comparison of anemia-associated parameters between the two groups of patients indicated that the observation group had significantly lower $\mathrm{Hb}$, PLT and MCV compared with the control group $(\mathrm{P}<0.05$; Fig. 3$)$.

Comparison of cardiac function indicators. In terms of cardiac function, the observation group had a significantly lower $\mathrm{CO}$,
SV and LVEF and higher LVEDd and LVESd compared with the control group $(\mathrm{P}<0.05$; Table II).

Correlations of cardiac function with serum inflammatory cytokines, oxidative stress and anemia. To analyze the association of cardiac dysfunction with inflammatory cytokines, oxidative stress or anemia, a correlation analysis was performed. It was indicated that the LVEF was negatively correlated with CRP $(\mathrm{r}=-0.636, \mathrm{P}<0.01$; Fig. 4A) and MDA $(\mathrm{r}=-0.705, \mathrm{P}<0.01$; Fig. 4B), but positively correlated with $\mathrm{Hb}$ levels ( $r=0.732$, $P<0.001$; Fig. 4C).

\section{Discussion}

Uremia, a chronic inflammatory disease, is generally considered as a reaction (in cellular, biochemical and other aspects) of the patient's body in response to renal failure, the major driving factors of which are a series of inflammatory cytokines and the most striking feature is chronic oxidation (10). Uremic patients frequently exhibit excessive inflammatory response and different degrees of anemia. Iron mobilization declines due to a markedly reduced production of erythropoietin. Furthermore, there is a certain degree of resistance to erythropoietin in uremic patients (11). The major causes of death in patients with uremia are cardiovascular disease-associated complications, as demonstrated by impaired myocardial cell systolic or diastolic function in uremic patients, which are caused by myocardial cell tissue fibrosis, ventricular enlargement and hypertrophy, pericarditis and impairment of various valve areas (12). Numerous studies have indicated that cardiac insufficiency in patients with uremia is mainly caused by non-specific pathological changes in various aspects rather than uremia alone (13). The most common causes of cardiac insufficiency in uremic patients include an elevation in blood pressure, increased heart volume and load, anemia of various severities, release of a large number of inflammatory cytokines and uremic toxins, as well as long-term oxidative stress (3). A recent study has suggested that CRP and IL may effectively reflect the degree of inflammation in uremic patients (14). It has been indicated that inflammation is a common cause of renal impairment and a major cause of morbidity and mortality of patients with kidney disease, and overactivation of the inflammatory response in uremic patients is closely linked to infection, toxin release and malnutrition (15). Consistent with these results, the present study indicated that inflammatory cytokines in uremic patients complicated with cardiac insufficiency were significantly higher than those in uremic patients without cardiac insufficiency. Furthermore, inflammatory cytokines were also negatively correlated with the LVEF, suggesting that the inflammation triggered by increased secretions of inflammatory cytokines may be a contributing factor in the development of cardiac insufficiency, implying that anti-inflammatories may be beneficial to improve cardiac function in uremic patients with cardiac insufficiency.

Oxidative stress, which causes lipid peroxidation, may contribute to increase the risk of atherosclerosis. Oxidative stress in patients with uremia refers to increased reactive oxygen species in the body. Recent study indicates that levels of lipid peroxidation products (malonaldehyde and 4-hydroxyalkenals) are significantly raised in the plasma of renal patients 
Table II. Comparison of cardiac function indicators.

\begin{tabular}{lccr}
\hline Relevant indicator & Observation group $(\mathrm{n}=43)$ & Control group $(\mathrm{n}=36)$ & P-value \\
\hline CO, 1 min (mean $\pm \mathrm{SD})$ & $3.73 \pm 0.82$ & $4.39 \pm 0.88$ & 0.025 \\
SV, $\mathrm{ml}($ mean $\pm \mathrm{SD})$ & $40.78 \pm 6.15$ & $51.90 \pm 5.69$ & 0.003 \\
LVEF, \% (n) & $32 \pm 7$ & $43 \pm 5$ & 0.039 \\
LVEDd, mm (mean $\pm \mathrm{SD})$ & $73.39 \pm 7.42$ & $69.58 \pm 8.03$ & 0.040 \\
LVESd, mm (mean $\pm \mathrm{SD})$ & $59.29 \pm 3.05$ & $48.75 \pm 4.28$ & 0.035 \\
\hline
\end{tabular}

CO, cardiac output; SV, cardiac stroke volume; LVEF, left ventricular ejection fraction; LVEDd, left ventricular end-diastolic diameter; LVESd, left ventricular end-systolic diameter.
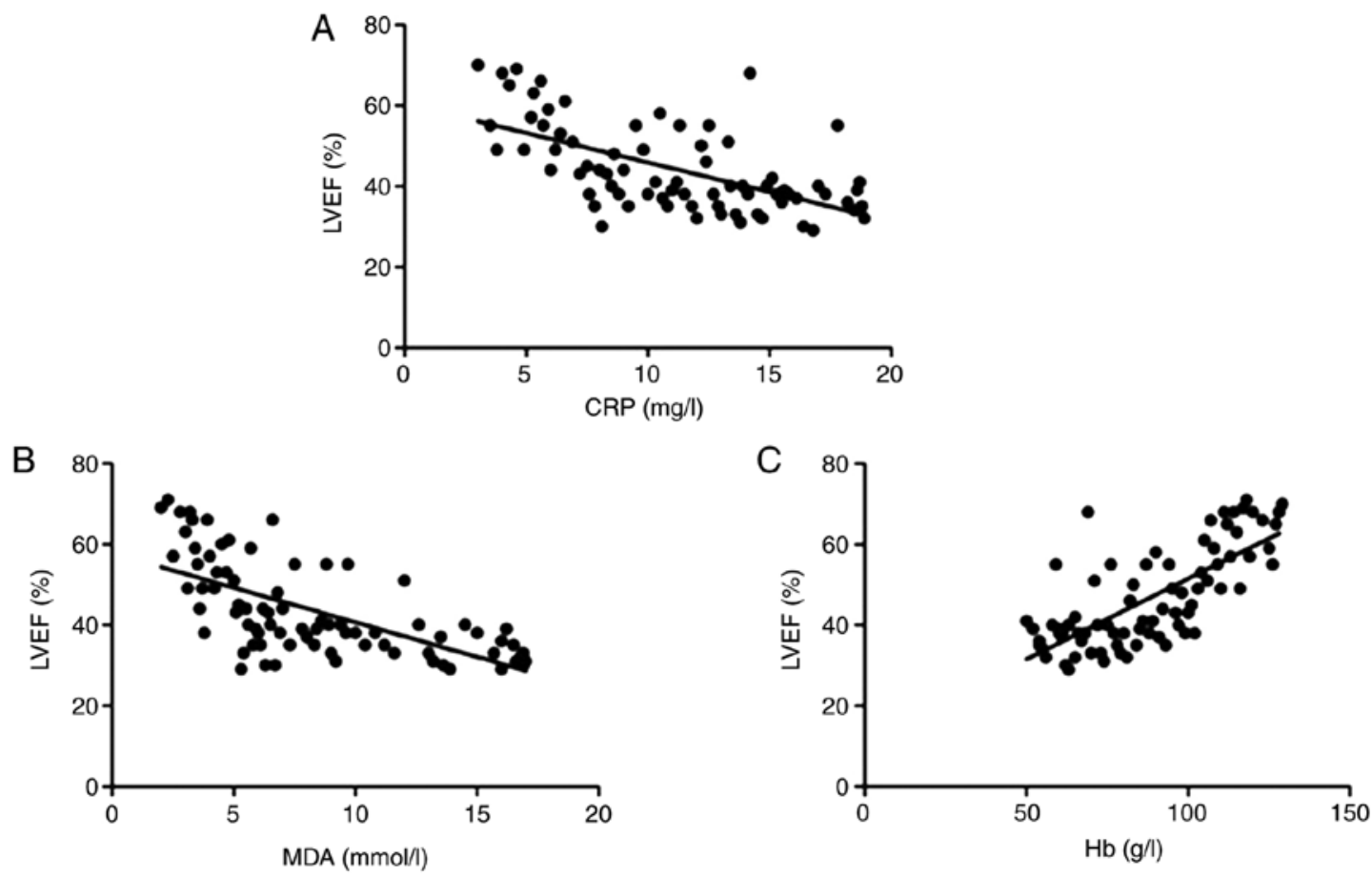

Figure 4. Correlation analysis of LVEF with (A) CRP, (B) MDA and (C) Hb. It was indicated that the LVEF was negatively correlated with CRP ( $\mathrm{r}=-0.636$, $\mathrm{P}<0.01)$ and MDA $(\mathrm{r}=-0.705, \mathrm{P}<0.01$; ), but positively correlated with $\mathrm{Hb}$ levels $(\mathrm{r}=0.732, \mathrm{P}<0.001)$. MDA, malondialdehyde; $\mathrm{CRP}$, C-reactive protein; Hb, hemoglobin; LVEF, left ventricular ejection fraction.

before dialysis and, although reduced after this treatment, the levels still remained above the normal range (16). Once there is a dynamic imbalance between oxidation and anti-oxidative processes in the body, the internal environment becomes unstable. Uremic patients suffer from disorder of the internal environment (i.e., oxidative stress) for a long time, causing damage to endothelial cell tissues (17). In general, disorder of lipid and calcium-phosphorus metabolism occurs in uremic patients, resulting in activated oxidation of fat and protein, and increased levels of oxidation products, including MDA in the patients' body (18). Oxidation products, including MDA and AOPP, are closely linked to atherosclerosis. Furthermore, increased levels of oxidation products in vivo may lead to impaired cardiovascular endothelial function (19). In accordance with these previous results, the present study indicated that uremic patients with cardiac insufficiency had significantly higher levels of oxidation products in comparison with those without cardiac insufficiency. On the other hand, patients with uremia suffer from anemia due to the reduction of erythropoietin, which is aggravated, as red blood cells have increased fragility and are more susceptible to rupture due to decreased levocarnitine and other substances in the body. Furthermore, anemia leads to insufficient blood supply to the myocardium, resulting in impaired cardiac function (19). The present study indicated that the degree of anemia in uremic patients with cardiac insufficiency was higher than in patients without cardiac insufficiency, and $\mathrm{Hb}$ was positively correlated with LVEF. A previous study evaluated the effect of left ventricular hypertrophy and deformation on cardiac function in patients with uremic cardiomyopathy by using the technology of two-dimensional speckle tracking imaging and the result indicated that the LVEF was decreased as cardiac function was reduced (20), which was consistent with the present study. However, limitations with this study are that the current data is based on a single-center study with a relatively small cohort. The correlation between cardiac insufficiency in 
uremic patients and inflammatory cytokines, oxidative stress and anemia requires to be further validated in a large number of samples from different regions in order to decrease the bias.

In conclusion, the present study indicated that cardiac insufficiency in patients with uremia is closely associated with inflammatory cytokines, oxidative stress and the degree of anemia, suggesting that inhibition of inflammation and oxidative stress or amelioration of anemia may improve cardiac function in uremic patients with cardiac insufficiency.

\section{Acknowledgements}

Not applicable.

\section{Funding}

No funding was received.

\section{Availability of data and materials}

The datasets used and/or analyzed during the current study are available from the corresponding author on reasonable request.

\section{Authors' contributions}

HZ substantially contributed to the experimentation and acquisition of data. LF designed experiments, performed data analysis and wrote the manuscript. HL and LT contributed to the conception of the study. JZ and DX helped perform the analysis with constructive discussions. JF contributed significantly to the data analysis and manuscript preparation. All authors read and approved the final manuscript.

\section{Ethics approval and consent to participate}

The present study was approved by the Ethics Committee of the Affiliated Hospital of North Sichuan Medical College (Nanchong, China; no. 20160102AHNSMCE) and informed consent from the subjects was obtained prior to the study.

\section{Patient consent for publication}

Not applicable.

\section{Competing interests}

The authors declare that they have no competing interests.

\section{References}

1. Foley RN, Parfrey PS and Sarnak MJ: Clinical epidemiology of cardiovascular disease in chronic renal disease. Am J Kidney Dis 32 (Suppl 3): S112-S119, 1998

2. Usberti M, Gerardi GM, Gazzotti RM, Benedini S, Archetti S, Sugherini L, Valentini M, Tira P, Bufano G, Albertini A, et al: Oxidative stress and cardiovascular disease in dialyzed patients. Nephron 91: 25-33, 2002
3. Himmelfarb J, Stenvinkel P, Ikizler TA and Hakim RM: The elephant in uremia: Oxidant stress as a unifying concept of cardiovascular disease in uremia. Kidney Int 62: 1524-1538, 2002.

4. Witko-Sarsat V, Friedlander $M$, Capeillère-Blandin C, Nguyen-Khoa T, Nguyen AT, Zingraff J, Jungers $P$ and Descamps-Latscha B: Advanced oxidation protein products as a novel marker of oxidative stress in uremia. Kidney Int 49: 1304-1313, 1996

5. Morena M, Cristol JP and Canaud B: Why hemodialysis patients are in a prooxidant state? What could be done to correct the pro/antioxidant imbalance. Blood Purif 18: 191-199, 2000.

6. Hirayama A, Nagase S, Gotoh M, Takemura K, Tomida C, Ueda A, Aoyagi K, Terao J and Koyama A: Hemodialysis does not influence the peroxidative state already present in uremia. Nephron 86: 436-440, 2000.

7. Miyata T, Ueda Y, Yamada Y, Izuhara Y, Wada T, Jadoul M, Saito A, Kurokawa K and van Ypersele de Strihou C: Accumulation of carbonyls accelerates the formation of pentosidine, an advanced glycation end product: Carbonyl stress in uremia. J Am Soc Nephrol 9: 2349-2356, 1998

8. Zimmermann J, Herrlinger S, Pruy A, Metzger T and Wanner C: Inflammation enhances cardiovascular risk and mortality in hemodialysis patients. Kidney Int 55: 648-658, 1999.

9. Riella MC: Malnutrition in dialysis: Malnourishment or uremic inflammatory response? Kidney Int 57: 1211-1232, 2000.

10. Steinberg D, Parthasarathy S, Carew TE, Khoo JC and Witztum JL: Beyond cholesterol. Modifications of low-density lipoprotein that increase its atherogenicity. N Engl J Med 320: 915-924, 1989.

11. Mak S, Lehotay DC, Yazdanpanah M, Azevedo ER, Liu PP and Newton GE: Unsaturated aldehydes including 4-OH-nonenal are elevated in patients with congestive heart failure. J Card Fail 6: 108-114, 2000.

12. Cracowski JL, Devillier P, Durand T, Stanke-Labesque F and Bessard G: Vascular biology of the isoprostanes. J Vasc Res 38: 93-103, 2001

13. Schettler V, Wieland E, Methe H,Schuff-Werner P and Müller GA: Oxidative stress during dialysis: Effect on free radical scavenging enzyme (FRSE) activities and glutathione (GSH) concentration in granulocytes. Nephrol Dial Transplant 13: 2588-2593, 1998.

14. Uchida K: Role of reactive aldehyde in cardiovascular diseases. Free Radic Biol Med 28: 1685-1696, 2000.

15. Helmke A and von Vietinghoff S: Extracellular vesicles as mediators of vascular inflammation in kidney disease. World $\mathbf{J}$ Nephrol 5: 125-138, 2016.

16. Fiorillo C, Oliviero C, Rizzuti G, Nediani C, Pacini A and Nassi P: Oxidative stress and antioxidant defenses in renal patients receiving regular haemodialysis. Clin Chem Lab Med 36: 149-153, 1998.

17. Canaud B, Cristol J, Morena M, Leray-Moragues H, Bosc J and Vaussenat F: Imbalance of oxidants and antioxidants in haemodialysis patients. Blood Purif 17: 99-106, 1999.

18. 19. Siems WG, Sommerburg O and Grune T: Erythrocyte free radical and energy metabolism. Clin Nephrol 53 (Suppl): S9-S17, 2000.

19. Siems W, Carluccio F, Grune T, Jakstadt M, Quast S, Hampl H and Sommerburg O: Elevated serum concentration of cardiotoxic lipid peroxidation products in chronic renal failure in relation to severity of renal anemia. Clin Nephrol 58 (Suppl 1): S20-S25, 2002.

20. Zheng Y, Yang X, Zhou Q, Huang Y, Zhang F and Wang L: Effect of left ventricular hypertrophy and deformation on cardiac function in patients with uremia cardiomyopathy by using quantitative speckle tracking technology. Zhong Nan Da Xue Xue Bao Yi Xue Ban 42: 400-405, 2017 (In Chinese).

(i) $\odot$ This work is licensed under a Creative Commons Attribution-NonCommercial-NoDerivatives 4.0 International (CC BY-NC-ND 4.0) License. 